\title{
QSPR study on the octanol/air partition coefficient of polybrominated diphenyl ethers by using molecular distance-edge vector index
}

\author{
Long Jiao ${ }^{1,2^{*}}$, Mingming $\mathrm{Gao}^{3}$, Xiaofei Wang ${ }^{1}$ and Hua $\mathrm{Li}^{2}$
}

\begin{abstract}
Background: The quantitative structure property relationship (QSPR) for octanol/air partition coefficient $\left(K_{\mathrm{OA}}\right)$ of polybrominated diphenyl ethers (PBDEs) was investigated. Molecular distance-edge vector (MDEV) index was used as the structural descriptor of PBDEs. The quantitative relationship between the MDEV index and the IgKOA Of PBDEs was modeled by multivariate linear regression (MLR) and artificial neural network (ANN) respectively. Leave one out cross validation and external validation was carried out to assess the predictive ability of the developed models. The investigated 22 PBDEs were randomly split into two groups: Group I, which comprises 16 PBDEs, and Group II, which comprises 6 PBDEs.

Results: The MLR model and the ANN model for predicting the $K_{\mathrm{OA}}$ of PBDEs were established. For the MLR model, the prediction root mean square relative error (RMSRE) of leave one out cross validation and external validation is 2.82 and 2.95, respectively. For the L-ANN model, the prediction RMSRE of leave one out cross validation and external validation is 2.55 and 2.69 , respectively.
\end{abstract}

Conclusion: The developed MLR and ANN model are practicable and easy-to-use for predicting the $K_{\mathrm{OA}}$ Of PBDEs. The MDEV index of PBDEs is shown to be quantitatively related to the $K_{\mathrm{OA}}$ of PBDEs. MLR and ANN are both practicable for modeling the quantitative relationship between the MDEV index and the $K_{\mathrm{OA}}$ of PBDEs. The prediction accuracy of the ANN model is slightly higher than that of the MLR model. The obtained ANN model shoud be a more promising model for studying the octanol/air partition behavior of PBDEs.

Keywords: QSPR, Polybrominated diphenyl ethers, Octanol/air partition coefficient, Molecular distance-edge vector index, Artificial neural network

\section{Background}

Polybrominated diphenyl ethers (PBDEs) are a series of organobromine compounds that have been widely used as flame retardant in a variety of products, such as building materials, electronics, furnishings, coatings, plastics, etc $[1,2]$. Although the production of some PBDEs has been restricted under the Stockholm Convention since 2010, PBDEs have already become ubiquitous pollutants in the environment. They have been detected in many environmental compartments, such as air, water, soil,

\footnotetext{
* Correspondence: mop@xsyu.edu.cn

${ }^{1}$ College of Chemistry and Chemical Engineering, Xi'an Shiyou University,

Xi'an 710065, People's Republic of China

${ }^{2}$ College of Chemistry and Materials Science, Northwest University, Xi'an

710069, People's Republic of China

Full list of author information is available at the end of the article
}

vegetations, animals and humans $[3,4]$. PBDEs have gained increasing attention because of their environmental persistence, bioaccumulation through the food chain, and potential risk to the human health $[1,5,6]$. PBDEs are lipophilic and semi-volatile compounds. The octanol/air partition of PBDEs may influence their fate, transport, and transformation in atmospheres [7-9]. The octanol/air partition coefficient $\left(K_{\mathrm{OA}}\right)$, which is defined as the ratio of solute concentration in air versus octanol when the octanol/air system is at equilibrium, is a key parameter for describing the octanol/air partition of PBDEs between the atmosphere and organic phases such as soil, aerosol, vegetation and animals. Thus, a quantitative study on the $K_{\mathrm{OA}}$ of PBDEs is of great importance to understand the environmental fate of PBDEs. Many efforts have been made to determine the $K_{\mathrm{OA}}$ of PBDEs 
[7,9-11]. However, determining the $K_{\mathrm{OA}}$ of PBDEs is always a hard work due to the complexity of analytical methods, lack of chemical standards and high cost of experiments [4,12-15]. Thus, the quantitative structureproperty relationship (QSPR) method, which is fast, easy-to-use and cost-effective [12,16,17], is always used to preliminary estimate the value of $K_{\mathrm{OA}}$ of PBDEs. Several QSPR models for the $K_{\mathrm{OA}}$ of PBDEs have been reported [12-15]. In these works, quantum chemical descriptors are used as the structural descriptor of PBDEs. However, developing a QSPR model based on quantum chemical descriptors is still a complex work, because the calculation and selection of structural descriptors are always time-consuming and complicated. It is still worthwhile to develop an easy-to-use QSPR model for the $K_{\mathrm{OA}}$ of PBDEs. Topological index is a kind of structural descriptor which has been widely used in the QSPR researches. It can effectively describe the structure of molecules without the detailed molecular orbital calculation and energy optimization. Topological index is useful because, despite its mathematical simplicity, it is able to differentiate molecules with different structures [18]. Therefore, the aim of our work is to investigate the QSPR model for the $K_{\mathrm{OA}}$ of PBDEs based on topological index. Molecular distance-edge vector (MDEV) index [19-21] was used as the structural descriptor of PBDEs. Multivariate linear regression (MLR) and artificial neural network (ANN) were employed to build the calibration model between the MDEV index and the $K_{\mathrm{OA}}$ of PBDEs.

\section{Results and discussion}

Firstly, the MDEV index of the investigated 22 PBDEs was calculated. The obtained MDEV index is presented in Table 1. As shown in the table, the value of MDEV index for different PBDE molecules is different. It is demonstrated that MDEV index can describe the structural differences among these molecules. Thus, it is reasonable to use MDEV index as structural descriptor to develop the QSPR model of PBDEs.

Secondly, two QSPR models were developed and investigated. One is MLR model and the other is L-ANN model. In order to assess the predictive ability of the developed models, two validation methods, leave one out cross validation and external validation, were conducted. The 22 PBDEs were randomly divided to two groups: Group I, which comprises 16 PBDEs, and Group II, which comprises 6 PBDEs (marked by asterisk in Tables 1 and 2).

\section{MLR model}

Generally, a simple model should always be chosen in preference to a complex model, if the latter does not fit the data better. Thus, we firstly investigate whether MLR can model the quantitative relationship between
Table 1 MDEV index of the investigated PBDEs

\begin{tabular}{|c|c|c|c|}
\hline No. & PBDE congeners & $\mu_{1}$ & $\mu_{2}$ \\
\hline 1 & 2 -monobro & 0 & 1.1111 \\
\hline $2^{*}$ & 3 -monobro & 0 & 1.0625 \\
\hline 3 & 2,4 -dibro & 0.0625 & 2.1511 \\
\hline 4 & 2,4' -dibro & 0.0204 & 2.1511 \\
\hline 5 & 2,6 -dibro & 0.0625 & 2.2222 \\
\hline $6^{*}$ & 3,4 -dibro & 0.1111 & 2.1025 \\
\hline 7 & 3,4' -dibro & 0.0156 & 2.1025 \\
\hline 8 & 4,4' -dibro & 0.0123 & 2.0800 \\
\hline 9 & 2,3,4 -tribro & 0.2847 & 3.2136 \\
\hline $10^{*}$ & 2,4,6 -tribro & 0.1875 & 3.2622 \\
\hline 11 & 2,4',6 -tribro & 0.1033 & 3.2622 \\
\hline 12 & 3,3', 4 -tribro & 0.1471 & 3.1650 \\
\hline 13 & 3,4,4' -tribro & 0.1391 & 3.1425 \\
\hline $14^{*}$ & $2,2^{\prime}, 4,4^{\prime}$-tetrabro & 0.2182 & 4.3022 \\
\hline 15 & $2,3^{\prime}, 4,4^{\prime}$-tetrabro & 0.2498 & 4.2536 \\
\hline 16 & $2,3^{\prime}, 4,6$-tetrabro & 0.2587 & 4.3247 \\
\hline 17 & $2,4,4^{\prime}, 6$-tetrabro & 0.2407 & 4.3022 \\
\hline $18^{*}$ & $3,3^{\prime}, 4,4^{\prime}$-tetrabro & 0.2862 & 4.2050 \\
\hline 19 & 2,2',3,3',4 -pentabro & 0.5478 & 5.3872 \\
\hline 20 & $2,2^{\prime}, 4,4^{\prime}, 5$ - pentabro & 0.4127 & 5.3647 \\
\hline 21 & 2,3',4,4',6 -pentabro & 0.4230 & 5.3647 \\
\hline $22^{*}$ & $2,2^{\prime}, 4,4^{\prime}, 5,5^{\prime}$-hexabro & 0.6276 & 6.4272 \\
\hline
\end{tabular}

*The PBDE congeners in the test set (see text).

the MDEV index and the $\lg K_{\mathrm{OA}}$ of these PBDEs. The MDEV index was used as independent variable and the $\lg K_{\mathrm{OA}}$ was used as dependent variable to develop the model.

Firstly, leave one out cross validation was carried out. In the leave one out cross validation, the $\lg K_{\mathrm{OA}}$ of all the samples in Group I was predicted in turn. The prediction procedure was performed 16 times. In each time, one sample was selected and used as the test set. The remaining 15 samples were used as training set to develop the regression model. The $\lg K_{\mathrm{OA}}$ of the selected sample (test set) was then predicted with the obtained regression model. The result of leave one out cross validation is listed in Table 2 . As shown in Table 2, the predicted $\lg K_{\mathrm{OA}}$ are in good agreement with the experimental $\lg K_{\mathrm{OA}}$. For the 16 samples of Group I, the prediction $R M S R E$ is 2.82 . In addition, the predicted $\lg K_{\mathrm{OA}}$ were plotted versus the experimental $\lg K_{\mathrm{OA}}$. The obtained plot is shown in Figure 1. The plot shows a linear relationship $\left(\lg K_{O A \text {,pred }}=0.9635 \lg K_{O A, \exp }+0.3573\right.$ with $\left.R=0.9769\right)$ between the predicted and experimental $\lg K_{\mathrm{OA}}$.

Subsequently, external validation was carried out to further assess the predictive ability of the MLR model. The regression model was developed by using all the 16 
Table 2 Experimental and predicted $\lg K_{\mathrm{OA}}$ of the investigated PBDEs

\begin{tabular}{|c|c|c|c|c|c|}
\hline \multirow[t]{2}{*}{ No. } & \multirow{2}{*}{$\begin{array}{l}\text { Experimental } \\
\lg K_{\mathrm{OA}}\end{array}$} & \multicolumn{2}{|c|}{ Predicted $\lg K_{\mathrm{OA}}$} & \multicolumn{2}{|c|}{ Relative error (\%) } \\
\hline & & MLR & ANN & MLR & ANN \\
\hline 1 & 7.24 & 7.56 & 7.45 & 4.42 & 3.59 \\
\hline $2^{*}$ & 7.36 & 7.38 & 7.40 & 0.27 & 0.54 \\
\hline 3 & 8.37 & 8.43 & 8.43 & 0.72 & 0.36 \\
\hline 4 & 8.47 & 8.46 & 8.45 & -0.12 & -0.12 \\
\hline 5 & 8.12 & 8.54 & 8.50 & 5.17 & 5.05 \\
\hline $6^{*}$ & 8.55 & 8.40 & 8.35 & -1.75 & -2.34 \\
\hline 7 & 8.57 & 8.39 & 8.41 & -2.10 & -1.63 \\
\hline 8 & 8.64 & 8.35 & 8.39 & -3.36 & -3.01 \\
\hline 9 & 9.49 & 9.22 & 9.33 & -2.85 & -2.42 \\
\hline $10^{*}$ & 9.02 & 9.53 & 9.44 & 5.65 & 4.66 \\
\hline 11 & 9.28 & 9.54 & 9.49 & 2.80 & 2.26 \\
\hline 12 & 9.61 & 9.34 & 9.37 & -2.81 & -2.81 \\
\hline 13 & 9.68 & 9.32 & 9.35 & -3.72 & -3.82 \\
\hline $14^{*}$ & 10.34 & 10.41 & 10.44 & 0.68 & 0.97 \\
\hline 15 & 10.49 & 10.34 & 10.37 & -1.43 & -1.05 \\
\hline 16 & 10.23 & 10.45 & 10.43 & 2.15 & 1.96 \\
\hline 17 & 10.13 & 10.47 & 10.42 & 3.36 & 2.96 \\
\hline $18^{*}$ & 10.7 & 10.27 & 10.30 & -4.02 & -3.74 \\
\hline 19 & 11.14 & 11.38 & 11.29 & 2.15 & 2.15 \\
\hline 20 & 11.28 & 11.35 & 11.36 & 0.62 & 0.27 \\
\hline 21 & 11.52 & 11.28 & 11.35 & -2.08 & -1.39 \\
\hline $22^{*}$ & 12.15 & 12.23 & 12.26 & 0.66 & 0.91 \\
\hline
\end{tabular}

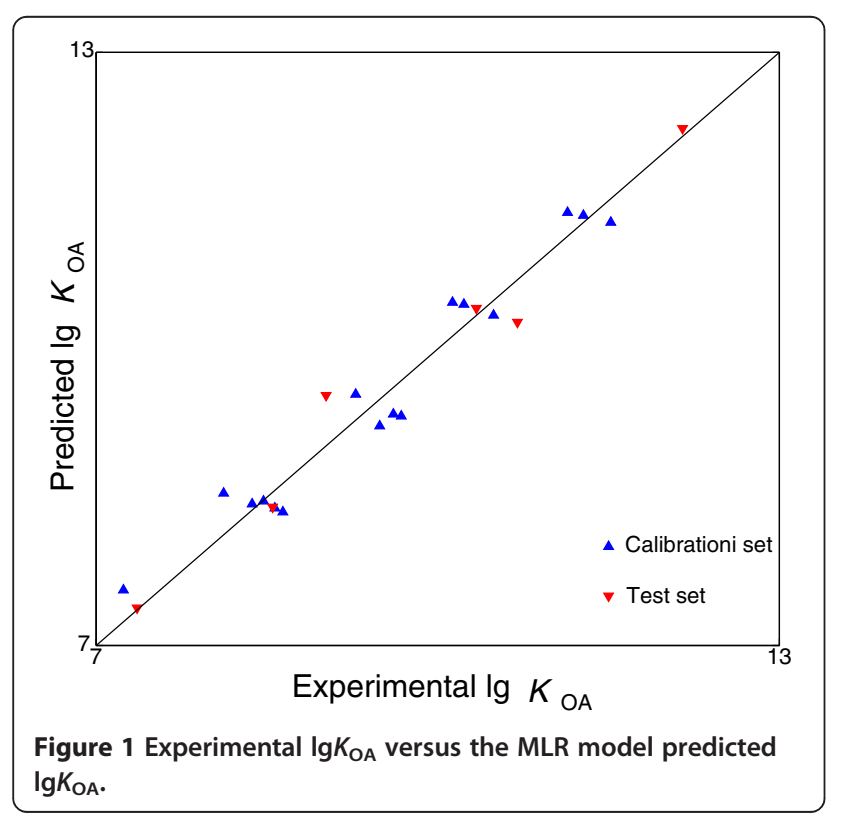

compounds in Group I. The obtained regression equation is:

$$
\lg K_{O A}=0.7598 \times \mu_{1}+0.9883 \times \mu_{2}-6.3470
$$

The $R$, Standard error of the estimate and $F$ value of the regression model is $0.9844,0.2340$ and 202.46, respectively. Then, the $\lg K_{\mathrm{OA}}$ of the six PBDEs in Group II was predicted by Equation 1. The prediction result is shown in Table 2 also. As shown in the table, the predicted $\lg K_{\mathrm{OA}}$ are still in good agreement with the experimental $\lg K_{\mathrm{OA}}$. The prediction RMSRE of the 6 PBDEs in Group II (marked by asterisk in Table 2) is 2.95 . The plot of the predicted $\lg K_{\mathrm{OA}}$ versus experimental $\lg K_{\mathrm{OA}}$ is presented in Figure 1. As shown in Figure 1, there is a linear relationship $\left(\lg K_{\mathrm{OA}, \text { pred }}=0.9721 \quad \lg K_{\mathrm{OA}, \exp }+0.2867\right.$ with $R=0.9836)$ between the predicted and experimental $\lg K_{\mathrm{OA}}$.

The results of leave one out cross validation and external validation demonstrates that the MDEV index is quantitatively related to the $K_{\mathrm{OA}}$ of PBDEs. The established MLR model can describe the quantitative relationship between the MDEV index and $K_{\mathrm{OA}}$ of PBDEs. Compared with the QSPR models reported in the references [12-15], the obtained MLR model shows comparative prediction accuracy. MDEV index can be generated easier than quantum chemical descriptors. Thus, the developed MLR model is a reliable and easy-to-use QSPR model for predicting the $K_{\mathrm{OA}}$ of PBDEs.

\section{L-ANN model}

L-ANN is an efficient and commonly used multivariate calibration method. Thus, we investigated whether a better model can be developed by using L-ANN appraoch. A 2-1 RBF-ANN (i.e. there are 2 nodes in the input layer and 1 node in the output layer) was used to model the quantitative relationship between the MDEV index and the $\lg K_{\mathrm{OA}}$. The MDEV index was used as the input variable and the $\lg K_{\mathrm{OA}}$ was used as the output variable.

Group I was still used to carry out leave one out cross validation. In the leave one out cross validation, the $\lg K_{\mathrm{OA}}$ of all the samples in Group I was predicted in turn. The prediction procedure was performed 16 times. In each time, one sample was selected and used as the test set. The remaining 15 samples were used as the calibration set to develop the network. Hence the 15 samples were randomly divided into a training set which includes 12 samples and a verification set which includes 3 samples. The $\lg K_{\mathrm{OA}}$ of the selected sample (test set) was then predicted with the obtained network. The result of leave one out cross validation is listed in Table 2. For the 16 samples of Group I, the prediction RMSRE is 2.55. The plot of the predicted $\lg K_{\mathrm{OA}}$ versus the experimental $\lg K_{\mathrm{OA}}$ is presented in Figure 2. The regression equation and correlation coefficient between the predicted and 


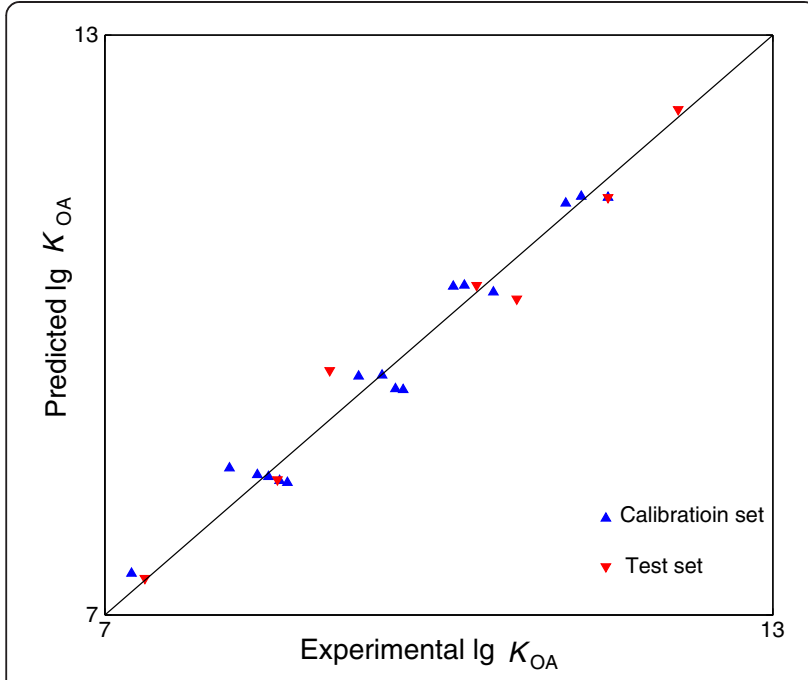

Figure 2 Experimental $\lg K_{\mathrm{OA}}$ versus the L-ANN model predicted $\lg K_{\mathrm{OA}}$.

experimental $\lg K_{\mathrm{OA}}$ is $\lg K_{\mathrm{OA}, \text { pred }}=0.9731 \lg K_{\mathrm{OA}, \text { exp }}+0.2640$ and 0.9812 respectively.

Subsequently, the external validation was carried out by using all the 22 PBDEs. An L-ANN model was developed from the 16 PBDEs in Group II. In the training procedure, the verification set comprises three randomly selected samples and the rest 13 samples were used as the training set. The $\lg K_{\mathrm{OA}}$ of the six PBDEs in Group I was then predicted with the obtained L-ANN model. The prediction result is presented in Table 2 also. The prediction RMSRE of the 6 PBDEs in Group II (marked by asterisk in Table 2) is 2.68 . The plot of the predicted $\lg K_{\mathrm{OA}}$ versus the experimental $\lg K_{\mathrm{OA}}$ is shown in Figure 2. There is a linear relationship $\left(\mathrm{g} K_{\mathrm{OA}}\right.$, pred $=0.9854$ $\lg K_{\mathrm{OA} \text {, exp }}+0.1535$ with $R=0.9864$ ) between the predicted and experimental $\lg K_{\mathrm{OA}}$. Obviously, the predicted $\lg K_{\mathrm{OA}}$ is in good agreement with the experimental $\lg K_{\mathrm{OA}}$. It is demonstrated that the quantitative relationship between the MDEV index and $\lg K_{\mathrm{OA}}$ of PBDEs has been modeled well by L-ANN. Compared with the QSPR models reported in the references [12-15], the obtained L-ANN model shows comparative accuracy in predicting the $\lg K_{\mathrm{OA}}$ of PBDEs. Obviously, it is a reliable and easy-to-use QSPR model for predicting the $\lg K_{\mathrm{OA}}$ of PBDEs. In addition, the prediction result of the L-ANN model is slightly better than the result of the MLR model. Therefore, the established L-ANN model should be a more promising model for studying the octanol/air partition behavior of PBDEs.

\section{Experimental}

\section{Data set}

The MDEV index was calculated according to the approach presented in section "Methods: MDEV index". The calculated MDEV index is listed in Table 1. The experimental $\lg K_{\mathrm{OA}}$ of the 22 PBDEs listed in Table 2 is taken from references [12].

Root mean square relative error (RMSRE) was calculated to indicate the prediction performance of the obtained models. RMSRE is defined as:

$$
\text { RMSRE }=\sqrt{\frac{\sum\left(R E_{i}\right)^{2}}{n}}
$$

where $R E_{i}$ is the relative error of the $i$ th sample, and $n$ is the number of samples.

\section{Software}

All the calculations were done with the subroutines developed under Matlab (Ver. 7.0). The computation was performed on a personal computer equipped with an i5-2450M processor. The used activation function of L-ANN is a linear function shown in Equation 5.

\section{Conclusion}

Two QSPR models for the octanol/air partition of PBDEs were developed by using MLR and L-ANN respectively. The results of leave one out cross validation and external validation indicate that the obtained MLR model and LANN model are practicable for predicting the $K_{\mathrm{OA}}$ of PBDEs. It is demonstrated that the MDEV index is quantitatively related to the $K_{\mathrm{OA}}$ of PBDEs. MDEV index can be generated easier than quantum chemical descriptors. Thus, using MDEV index as structural descriptor is more convenient than using quantum chemical descriptor when developing the QSPR model for the $K_{\mathrm{OA}}$ of PBDEs. In addition, the result demonstrates MLR and L-ANN are both practicable for modeling the quantitative relationship between the MDEV index and $K_{\mathrm{OA}}$ of PBDEs. Compared with the established MLR model, the obtained L-ANN model shows slightly higher prediction accuracy. The obtained L-ANN model should be a more promising model for studying the octanol/air partition behavior of PBDEs.

\section{Methods \\ MDEV index}

In the calculation of MDEV index, a molecule is regarded as a geometric graph. Each non-hydrogen atom is regarded as a point and each chemical bond is regarded as an edge. The molecular structure of PBDEs can be encoded by the MDEV index of bromine atoms and benzene rings. If the relative electronegative of each bromine atom and benzene ring is defined as 1 , the MDEV index of PBDEs can be defined as Equation 3:

$$
M_{k l}=\sum_{j \geq i} \frac{1}{d_{i k, j l}^{2}}
$$

$(k, l=1,2$ and $l \geq k)$ 
where $k$ and $l$ denote the type of an atom $(k=1$ or $l=1$ denotes the bromine atom, and $k=2$ or $l=2$ denotes the benzene ring); $i$ and $j$ are the coding number of series number of a bromine atom or benzene ring in the molecular skeleton graph. In addition, $i$ and $j$ belong to the $k$ th and $l$ th type respectively. The $d_{i k, j l}$ means the shortest relative distance between the $i$ th and $j$ th atom. For example, $d_{i 1, j 1}$ denotes the nearest relative distance between the $i$ th and $j$ th bromine atom. The relative bond length between the two adjacent non-hydrogen atoms is defined as $d=1$. According to Equation 3, there are three elements, $M_{11}, M_{12}$ and $M_{22}$, in the MDEV index for a PBDE molecule. The three elements are usually noted as $\mu_{1}, \mu_{2}$ and $\mu_{3}$ respectively. For example, the MDEV index of 2,2,4,4'-PBDE should be calculated as follows:

$$
\begin{aligned}
\mu_{1}=M_{11}= & \left(\frac{1}{4}\right)^{2}+\left(\frac{1}{5}\right)^{2}+\left(\frac{1}{7}\right)^{2}+\left(\frac{1}{7}\right)^{2}+\left(\frac{1}{9}\right)^{2}+\left(\frac{1}{4}\right)^{2}=0.2182 \\
\mu_{2}=M_{12}= & \left(\frac{1}{1}\right)^{2}+\left(\frac{1}{3}\right)^{2}+\left(\frac{1}{1}\right)^{2}+\left(\frac{1}{3}\right)^{2}+\left(\frac{1}{1}\right)^{2}+\left(\frac{1}{4}\right)^{2} \\
& +\left(\frac{1}{1}\right)^{2}+\left(\frac{1}{4}\right)^{2}=4.3022 \\
\mu_{3}= & M_{22}=\left(\frac{1}{1}\right)^{2}=1
\end{aligned}
$$

Obviously, the $M_{22}$ of each PBDE is equal to 1 . Thus, $\mu_{1}$ and $\mu_{2}$ were used to describe the structure of PBDEs.

\section{Artificial neural network}

The theory of ANN has been elaborated in a lot of articles [21-28]. Hence, only a brief outline of ANN is presented here.

ANN is a multivariate calibration method capable of modeling complex functions. The basic processing unit of ANN is the neuron (node). An artificial neural network comprises a number of neurons organized in different layers. Linear artificial neural network (L-ANN) [22-25] is a neural network having no hidden layers, but an output layer with fully linear neurons (that is, linear neurons with linear activation function). It is the simplest artificial neural network. In L-ANN, the neurons between the input and output layers fully connect, while the neurons in the same layer do not. Figure 3 illustrates the basic architecture of the used L-ANN.

In Figure 3, $x_{1}$ and $x_{2}$ are the input variables; $y_{1}$ and $w_{1}$ denotes the output variables and the element of connection weight matrix $\mathbf{W}$ respectively; $b_{1}$ is the bias vector. The symbol fact( ) means the activation function. Previous to training procedure, the input and output variables are normalized. When the network is executed, it multiplies the input variables by the weights matrix, and then adds the bias vector. The post synaptic potential

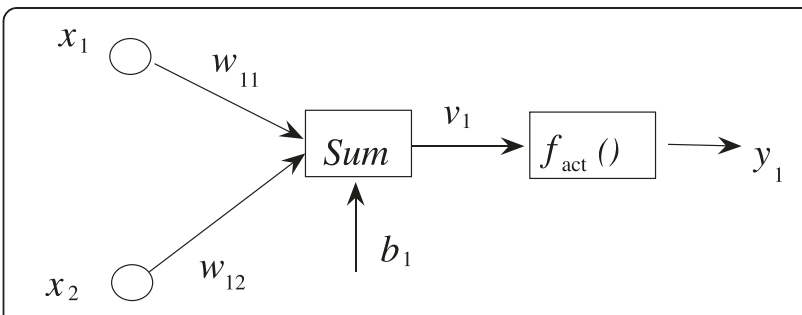

Input layer

Output layer

Figure 3 Architecture of linear artificial neural network.

(PSP) function of the neuron can be described as Equation 5:

$$
v_{\mathrm{j}}=\sum_{i=1}^{n} x_{\mathrm{i}} w_{\mathrm{ij}}+b_{\mathrm{j}}
$$

Generally, the activation function used in L-ANN is a linear function which can be described as:

$$
y_{j}=v_{j}
$$

Because there are no non-linear functions and hidden neurons in the network, L-ANN is ideal for dealing with linear problems. Actually, training a linear network means finding the optimal setting for the weight matrix W to minimize the root mean squared error (RMSE) of calibration set. In order to achieve this aim, the known samples which are used as calibraion set are generally divided into two parts: a training set and a verification set. The training set was used to calculate and adjust the network weights. The verification set was used to track the network's error performance, to identify the best network, and to stop training. The training should be stopped once deterioration in the verification error is observed. The optimal network parameters were selected according to the RMSE of verification set. The overfitting and over-learning can be effectively avoided in this way. Although the verification set is used to identify the best network, actually, training algorithms do not use the verification set to adjust network weights. Standard pseudo-inverse linear optimization algorithm [22] is usually used to train the network. This algorithm uses the singular value decomposition technique to calculate the pseudo-inverse of the matrix needed to set the weights in a linear output layer, so as to find the least mean squared solution. Essentially, it guarantees to reach the optimal setting for the weights in the linear layer.

The main difference between MLR and L-ANN is the optimization algorithm. In MLR, the aim of least square algorithm is to minimize the sum of squared residuals of the training set. As for L-ANN, the aim of training algorithm is to minimize the RMSE of verification set [22]. 


\section{Leave one out cross validation}

Leave one out cross validation [29] is a commonly used algorithm for estimating predictive performance of a multivariable calibration model. Usually, practical calibration experiments have to be based on a limited set of available samples. The idea behind the leave one out cross validation algorithm is to predict the property value of each sample in turn with the calibration model which is developed with the other samples. When applying the algorithm to a dataset with $N$ samples, the calibration modeling is performed $N$ times, each time using $(N-1)$ samples for modeling and one sample for testing. Thus, the procedure of leave one out cross validation can be divided into $N$ segment. In each segment $i(i=1, \ldots, N)$, there are three steps: (1) taking sample $i$ out as temporary 'test set', which is not used to develop the calibration model, (2) developing the calibration model with the remaining $(N-1)$ samples, (3) testing the developed model with sample $i$, calculating and storing the prediction error of the sample.

\section{External validation}

External validation [26,30] is a algorithm which has been generally applied to estimating predictive performance of calibration models. When utilizing the algorithm, working dataset is split into two subsets: a calibration set, which is used to establish the calibration model, and a test set, which is employed to assess the predictive ability of the established calibration model. Herein, test set is designed to give an independent assessment of the predictive performance of the assed model. It is not used in establishing the calibration mdoel at all, and hence is independent of the calibration set. Generally, the samples in calibration set and test set are randomly selected from the working dataset.

\section{Competing interests}

The authors declare that they have no competing interests.

\section{Authors' contributions \\ $\downarrow J$ conceived of the study, carried out the calculation of structural descriptor and the model building, draft the manuscript. MMG and XFW participated in the design of the study and performed the statistical analysis, HL coordination and helped to draft the manuscript. All authors read and approved the final manuscript.}

\section{Acknowledgments \\ The work was supported by the National Natural Science Foundation of China No. 21305108, the Project Supported by Natural Science Basic Research Plan in Shaanxi Province of China (Program No. 2014JM2039), and the Innovative Research Team of Xi'an Shiyou University.}

\section{Author details}

${ }^{1}$ College of Chemistry and Chemical Engineering, Xi'an Shiyou University, Xi'an 710065, People's Republic of China. ${ }^{2}$ College of Chemistry and Materials Science, Northwest University, Xi'an 710069, People's Republic of China. ${ }^{3}$ No.203 Research Institute of Nuclear industry, Xianyang 712000, People's Republic of China.
Received: 5 March 2014 Accepted: 4 June 2014

Published: 10 June 2014

\section{References}

1. Anderson HA, Imma P, Knobeloch L, Turyk M, Mathew J, Buelow C, Persky V: Polybrominated diphenyl ethers (PBDE) in serum: Findings from a US cohort of consumers of sport-caught fish. Chemosphere 2008, 73:187-194.

2. EPA: America's children and the environment. 2014. http://www.epa.gov/ ace/pdfs/Biomonitoring-PBDEs.pdf.

3. Secretariat of the Stockholm Convention: Listing of POPs in the Stockholm Convention. 2014. http://chm.pops.int/TheConvention/ThePOPs/Listingof POPs/tabid/2509/Default.aspx.

4. Yue CY, Li LY: Filling the gap: estimating physicochemical properties of the full array of polybrominated diphenyl ethers (PBDEs). Environ Pollut 2013, 180:312-323.

5. Wang YW, Zhao CY, Ma WP, Liu HX, Wang T, Jiang GB: Quantitative structure-activity relationship for prediction of the toxicity of polybrominated diphenyl ether (PBDE) congeners. Chemosphere 2006, 64:515-524.

6. Watkins DJ, McClean MD, Fraser AJ, Weinberg J, Stapleton HM, Webster TF: Associations between PBDEs in office air, dust, and surface wipes. Environ Int 2013, 59:124-132.

7. Harner T, Shoeib M: Measurements of octanol-air partition coefficients (KOA) for polybrominated diphenyl ethers (PBDEs): predicting partitioning in the environment. J Chem Eng Data 2002, 47:228-232.

8. Mizukawa K, Takada H, Takeuchi I, Ikemoto T, Omori K, Tsuchiya K: Bioconcentration and biomagnification of polybrominated diphenyl ethers (PBDEs) through lower-trophic-level coastal marine food web. Mar Pollut Bull 2009, 58:1217-1224.

9. Wania F, Lei YD, Harner T: Estimating octanol-air partition coefficients of nonpolar semivolatile organic compounds from gas chromatographic retention times. Anal Chem 2002, 74:3476-3483.

10. Han SY, Liang C, Qiao JQ, Lian HZ, Ge X, Chen HY: A novel evaluation method for extrapolated retention factor in determination of n-octanol/ water partition coefficient of halogenated organic pollutants by reversed-phase high performance liquid chromatography. Anal Chim Acta 2012, 713:130-135.

11. Cetin B, Odabasi M: Atmospheric concentrations and phase partitioning of polybrominated diphenyl ethers (PBDEs) in Izmir, Turkey. Chemosphere 2008, 71:1067-1078.

12. Xu HY, Zou JW, Yu QS, Wang YH, Zhang JY, Jin HX: QSPR/QSAR models for prediction of the physicochemical properties and biological activity of polybrominated diphenyl ethers. Chemosphere 2007, 66:1998-2010.

13. Wang ZY, Zeng XL, Zhai ZC: Prediction of supercooled liquid vapor pressures and n-octanol/air partition coefficients for polybrominated diphenyl ethers by means of molecular descriptors from DFT method. Sci Total Environ 2008, 389:296-305.

14. Chen JW, Harner T, Yang P, Quan X, Chen S, Schramm KW, Kettrup A: Quantitative predictive models for octanol-air partition coefficients of polybrominated diphenyl ethers at different temperatures. Chemosphere 2003, 51:577-584.

15. Papa E, Kovarich S, Gramatica P: Development, validation and inspection of the applicability domain of QSPR models for physicochemical properties of polybrominated diphenyl ethers. QSAR Comb Sci 2009, 28:790-796

16. Nandi S, Monesi A, Drgan V, Merzel F, Novič M: Quantitative structureactivation barrier relationship modeling for Diels-Alder ligations utilizing quantum chemical structural descriptors. Chem Cent J 2013, 7:171.

17. Steffen A, Apostolakis J: On the ease of predicting the thermodynamic properties of beta-cyclodextrin inclusion complexes. Chem Cent J 2007, 1:29.

18. Gutman I, Tosovic J: Testing the quality of molecular structure descriptors. Vertex-degree based topological indices. J Serb Chem Soc 2013, 78:805-810.

19. Liu HH, Xiao X, Qin J, Liu YM: Study on structural characteristics and QSPR of polychlorinated biphenyls Isomers (PCBs). J Chongqing Inst Tech (In Chinese) 2005, 19:67-70.

20. Liu SS, Liu HL, Xia ZN, Cao CZ, Li ZL: Molecular distance-edge vector $(\mu)$ : an extension from alkanes to alcohols. J Chem Inf Comput Sci 1999, 39:951-957.

21. Yin CS, Guo WM, Lin T, Liu SS, Fu RQ, Pan ZX, Wang LS: Application of wavelet neural network to the prediction of gas chromatographic retention indices of alkanes. J Chin Chem Soc 2001, 48:739-749. 
22. Statsoft: Model extremely complex functions neural networks. 2013. http://www.statsoft.com/textbook/neural-networks.

23. Yin CS, Shen Y, Liu SS, Yin QS, Guo WM, Pan ZX: Simultaneous quantitative UV spectrophotometric determination of multicomponents of amino acids using linear neural network. Comput Chem 2001, 25:239-243.

24. Zhang WJ, Zhong $X Q$, Liu GH: Recognizing spatial distribution patterns of grassland insects: neural network approaches. Stoch Environ Res Risk Assess 2008, 22:207-216.

25. Zhang YX, Li H, Hou AX, Havel J: Artificial neural networks based on principal component analysis input selection for quantification in overlapped capillary electrophoresis peaks. Chemometr Intell Lab Syst 2006, 82:165-175.

26. Jalali-Heravi M, Garkani-Nejad Z: Prediction of electrophoretic mobilities of alkyl- and alkenylpyridines in capillary electrophoresis using artificial neural networks. J Chromatogr A 2002, 971:207-215.

27. Fatemi MH, Baher E: Quantitative structure-property relationship modelling of the degradability rate constant of alkenes by $\mathrm{OH}$ radicals in atmosphere. SAR QSAR Environ Res 2009, 20:77-90.

28. Abdollahi Y, Zakaria A, Abbasiyannejad M, Masoumi HRF, Moghaddam MG Matori KA, Jahangirian $\mathrm{H}$, Keshavarzi A: Artificial neural network modeling of p-cresol photodegradation. Chem Cent J 2013, 7:96.

29. Martens HA, Dardenne P: Validation and verification of regression in small data sets. Chemometr Intell Lab Syst 1998, 44:99-121.

30. Yin CS, Shen Y, Liu SS, Yin QS, Guo WM, Pan ZX: Simultaneous quantitative UV spectrophotometric determination of multicomponents of amino acids using linear neural network. Compu Chem 2001, 25:239-243.

doi:10.1186/1752-153X-8-36

Cite this article as: Jiao et al:: QSPR study on the octanol/air partition coefficient of polybrominated diphenyl ethers by using molecular distance-edge vector index. Chemistry Central Journal 2014 8:36.

\section{Publish with ChemistryCentral and every scientist can read your work free of charge \\ "Open access provides opportunities to our colleagues in other parts of the globe, by allowing anyone to view the content free of charge." \\ W. Jeffery Hurst, The Hershey Company. \\ - available free of charge to the entire scientific community \\ - peer reviewed and published immediately upon acceptance \\ - cited in PubMed and archived on PubMed Central \\ - yours - you keep the copyright \\ Submit your manuscript here: \\ http://www.chemistrycentral.com/manuscript/<smiles>c1ccccc1</smiles> \\ Chemistry Central}

